Published in final edited form as:

Science. 2019 March 01; 363(6430): 993-998. doi:10.1126/science.aat7186.

\title{
Epithelial endoplasmic reticulum stress orchestrates a protective IgA response
}

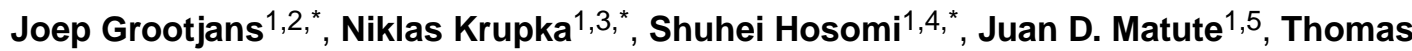 \\ Hanley ${ }^{1}$, Svetlana Saveljeva ${ }^{6}$, Thomas Gensollen ${ }^{1}$, Jarom Heijmans ${ }^{7}$, Hai Li $^{3}$, Julien P. \\ Limenitakis $^{3}$, Stephanie C. Ganal-Vonarburg ${ }^{3}$, Shengbao Suo ${ }^{8}$, Adrienne M. Luoma ${ }^{9}$, \\ Yosuke Shimodaira ${ }^{10}$, Jinzhi Duan ${ }^{1}$, David Q. Shih ${ }^{10}$, Margaret E. Conner ${ }^{11}$, Jonathan N. \\ Glickman $^{12}$, Gwenny M. Fuhler ${ }^{13}$, Noah W. Palm ${ }^{14}$, Marcel R. de Zoete ${ }^{15}$, C. Janneke van \\ der Woude ${ }^{13}$, Guo-Cheng Yuan ${ }^{8}$, Kai W. Wucherpfennig ${ }^{9}$, Stephan R. Targan ${ }^{10}$, Philip \\ Rosenstiel $^{16}$, Richard A. Flavell ${ }^{14,17}$, Kathy D. McCoy ${ }^{18}$, Andrew J. Macpherson ${ }^{3}$, Arthur \\ Kaser $^{6}$, and Richard S. Blumberg ${ }^{1, \dagger}$
}

1Division of Gastroenterology, Department of Medicine, Brigham and Women's Hospital, Harvard Medical School, 75 Francis Street, Boston, MA 02115, USA. ${ }^{2}$ Amsterdam University Medical Center, University of Amsterdam, Department of Gastroenterology and Hepatology and Tygat Institute for Liver and Intestinal Research, Meibergdreef 9, Amsterdam, Netherlands. ${ }^{3}$ Maurice Müller Laboratories (DBMR), Universitätsklinik für Viszerale Chirurgie und Medizin Inselspital, Murtenstrasse 35, University of Bern, 3010 Bern, Switzerland. " Department of Gastroenterology, Osaka City University Graduate School of Medicine, 1-4-3, Asahi-machi, Abeno-ku, Osaka 545-8585, Japan. ${ }^{5}$ Division of Neonatology, Department of Pediatrics, Massachusetts General Hospital, Harvard Medical School, Boston, MA 02114, USA. ${ }^{6}$ Division of Gastroenterology and Hepatology, Department of Medicine, University of Cambridge, Cambridge CB2 0QQ, UK. ${ }^{7}$ Amsterdam University Medical Center, University of Amsterdam, Department of Internal Medicine, Tygat Institute for Liver and Intestinal Research, Meibergdreef 9, Amsterdam, Netherlands. ${ }^{8}$ Department of Biostatistics and Computational Biology, Dana-Farber Cancer Institute, Boston, MA 02215, USA. ${ }^{9}$ Department of Cancer Immunology and Virology, DanaFarber Cancer Institute, 450 Brookline Avenue, Boston, MA 02215, USA. ${ }^{10} \mathrm{~F}$. Widjaja Foundation, Inflammatory Bowel and Immunobiology Research Institute, Cedars-Sinai Medical Center, Los Angeles, CA 90048, USA. ${ }^{11}$ Department of Molecular Virology and Microbiology, Baylor College

\footnotetext{
${ }^{\dagger}$ Corresponding author. rblumberg@bwh.harvard.edu.

Author contributions: J.G., N.K., S.H., and R.S.B. conceived, designed and interpreted the experiments; J.G., N.K., S.H., J.D.M., T.H., S.Sa., T.G., H.L., J.P.L., S.C.G.-V., S.Su., A.M.L., Y.S., J.D., G.M.F., N.W.P., and M.R.d.Z. carried out the experiments; J.N.G., P.R., R.A.F., K.D.M., A.J.M., and A.K. aided with the interpretation of the data; and J.G., N.K., and R.S.B. wrote the manuscript. All authors were involved in critical revision of the manuscript for important intellectual content.

*These authors contributed equally to this work.

Competing interests: K.W.W. serves on the scientific advisory board of TCR2 Therapeutics, T-Scan Therapeutics, and Nextechinvest and receives sponsored research funding from Astellas Pharma, Bristol-Myers Squibb, and Novartis.

Data and materials availability: Processed and raw data of the high-throughput sequencing experiments can be downloaded from NCBI Gene Expression Omnibus (GSE124561 and GSE124562). Pigr knockout mice were obtained under a materials transfer agreement with the University of California, San Diego (UCSD). All other data needed to evaluate the conclusions in this paper are present either in the main text or the supplementary materials.
}

SUPPLEMENTARY MATERIALS

www.sciencemag.org/content/363/6430/993/supp1/DC1 
of Medicine, Houston, TX 77030, USA. ${ }^{12}$ Department of Pathology, Beth Israel Deaconess Medical Center, Harvard Medical School, Boston, MA 02215, USA. ${ }^{13}$ Department of Gastroenterology and Hepatology, Erasmus MC, University Medical Center, Rotterdam, Netherlands. ${ }^{14}$ Department of Immunobiology, Yale University School of Medicine, New Haven, CT 06519, USA. ${ }^{15}$ Department of Infectious Diseases and Immunology, Faculty of Veterinary Medicine, Utrecht University, Utrecht, Netherlands. ${ }^{16}$ Institute of Clinical Molecular Biology, Christian-Albrechts-University Kiel, Rosalind-Franklin-Str. 12, 24105 Kiel, Germany. ${ }^{17}$ Howard Hughes Medical Institute, Yale University School of Medicine, New Haven, CT 06519, USA. ${ }^{18}$ Department of Physiology and Pharmacology, University of Calgary, Calgary, Alberta, Canada.

\section{Abstract}

Immunoglobulin $\mathrm{A}(\operatorname{Ig} \mathrm{A})$ is the major secretory immunoglobulin isotype found at mucosal surfaces, where it regulates microbial commensalism and excludes luminal factors from contacting intestinal epithelial cells (IECs). IgA is induced by both T cell-dependent and -independent (TI) pathways. However, little is known about TI regulation. We report that IEC endoplasmic reticulum (ER) stress induces a polyreactive IgA response, which is protective against enteric inflammation. IEC ER stress causes TI and microbiota-independent expansion and activation of peritoneal B1b cells, which culminates in increased lamina propria and luminal $\operatorname{IgA}$. Increased numbers of $\operatorname{IgA}$ producing plasma cells were observed in healthy humans with defective autophagy, who are known to exhibit IEC ER stress. Upon ER stress, IECs communicate signals to the peritoneum that induce a barrier-protective TI IgA response.

The intestinal epithelium is continuously confronted with potentially deleterious environmental stimuli (1). These exposures and the underlying secretory burden of intestinal epithelial cells (IECs) are challenging for this cell type. Thus, endoplasmic reticulum (ER) stress and the accompanying unfolded protein response (UPR) are commonly observed in IECs under homeostasis (2) and increased in inflammatory bowel disease (IBD) $(3,4)$. In IBD, ER stress in the IEC can serve as a nidus for spontaneous microbiota-dependent ileitis. This can be seen in mice with an IEC-restricted deletion of the important UPR effector molecule X-box binding protein $1\left(X b p 1^{\Delta \mathrm{IEC}}\right)(3,5)$. It is unknown, however, whether IECassociated ER stress can also elicit barrier-protective immune responses.

We observed higher numbers of immunoglobulin $\mathrm{A}$-positive $\left(\operatorname{Ig} \mathrm{A}^{+}\right)$plasma cells $\left(\mathrm{CD} 45^{+}\right.$ $\mathrm{CD}^{-}{ }^{-} \mathrm{IgA}^{+} \mathrm{B} 220^{-}$) in small-intestinal lamina propria (SI LP) and higher concentrations of ileal tissue IgA in $X b p 1^{\Delta \mathrm{IEC}}$ mice than in littermate $X b p 1^{\mathrm{fl} / \mathrm{fl}}$ controls (Fig. $1, \mathrm{~A}$ and B, and fig. S1). Secretory $\operatorname{IgA}(\operatorname{sg} A)$, which functions to protect the mucosa by coating and entrapping commensal and colitogenic bacteria (6) and excluding intraluminal factors from IEC contact $(7,8)$, was also increased in the lumen (Fig. 1C). This was associated with increased circulating IgA concentrations as early as 6 weeks of age (Fig. 1D), before the emergence of spontaneous inflammation in $X b p 1^{\Delta \mathrm{IEC}}$ mice. No other Ig isotypes were increased in the SI (fig. S2A) or sera (fig. S2B) of $X b p 1^{\Delta 1 E C}$ mice. The increased number of Ig $\mathrm{A}^{+}$cells in $X b p 1^{\Delta \mathrm{IEC}}$ mice accumulated around SI crypts (Fig. 1, E and F), where ER stress $(5,9)$ and basal plasmacytosis, a feature of IBD (10), frequently occur. 
$X b p 1$ deletion in IECs results in UPR activation, including the ER-stress sensor inositolrequiring enzyme 1 a (IRE1a) (11). Double conditional knockout mice lacking both IRE1a and XBP1 in IECs (Ern1/Xbp $\left.1^{\Delta \mathrm{IEC}}\right)$ showed no increase in $\mathrm{SI} \mathrm{IgA}{ }^{+}$cell numbers compared with $E r n 1 / X b p 1^{f l / f l}$ controls (fig. S3), indicating that IRE1a is an important mediator of the $\operatorname{IgA}$ response. We extended these observations to an inducible IEC-specific knockout of the ER-stress sensor glucose related protein 78 (GRP78) (12). Grp $78^{\text {T- } \Delta \mathrm{IEC}}$ mice exhibited a rapid increase in $\mathrm{SI} \mathrm{IgA}^{+}$plasma cells by 3 days after Grp 78 deletion (Fig. 1G). Conversely, treatment of $X b p 1^{\Delta \mathrm{IEC}}$ mice with the chemical chaperone tauroursodeoxycholic acid (TUDCA) (13) reduced IEC ER stress (fig. S4) and prevented the IgA response in the SI LP (Fig. 1H) and plasma (Fig. 1I).

We next generated $I g h a^{--} X b p 1^{\Delta \mathrm{IEC}}$ mice and $I g h a^{--} X b p I^{\mathrm{fl} / \mathrm{fl}}$ controls, which lack IgA. Consistent with previous studies (3), Xbp $1^{\Delta \mathrm{IEC}}$ mice developed spontaneous ileitis, which was unchanged under conditions of IgA deficiency (Fig. 1J). However, inflammation in $I g h a^{-l-} X b p 1^{\Delta \mathrm{IEC}}$ mice significantly extended proximally into the jejunum [Fig. $1 \mathrm{~K}$ and fig. S5, histology score and hematoxylin and eosin (H\&E), respectively], suggesting that IEC ER stress-induced IgA $\mathrm{A}^{+}$plasma cells protect from inflammation. Like humans with selective IgA deficiency (14), Igha ${ }^{-1}$ mice exhibited a compensatory increase of $\mathrm{LPIgM}^{+}$plasma cell numbers that was further increased with IEC ER stress (Igha ${ }^{-1} X b p 1^{\mathrm{AIEC}}$, Fig. 1L). We thus generated B cell-deficient $X b p 1^{\Delta \mathrm{IEC}}$ mice ( $\left.\mu M T X b p 1^{\mathrm{\Delta IEC}}\right)$, which lack intestinal LP plasma cells [IgA immunohisto-chemistry (IHC) images shown in fig. S6]. These animals showed no significant worsening of inflammation in either the jejunum or ileum compared with $I g h a^{--} X b p 1^{\Delta \mathrm{IEC}}$ controls (Fig. $1 \mathrm{~K}$ and fig. S5, histology scores and H\&E, respectively), indicating that in mice, compensatory IgM did not contribute to protection. This was likely due to its relatively low concentrations compared with those of IgA (fig. S7), a reduced ability of IgM to bind several typical IgA targets (15), and/or differences in secretory $\operatorname{IgM}(\mathrm{sIgM})$ function in mice compared with that in humans (16). Furthermore, the increased numbers of IgA ${ }^{+}$plasma cells in $X b p 1^{\Delta \mathrm{IEC}}$ mice were not due to increased concentrations of interleukin-10 (IL-10), which can be produced by B cells (17). SI tissue from $\mu M T X b p 1^{\Delta \mathrm{IEC}}$ and $X b p 1^{\Delta I E C}$ mice or $X b p 1^{\Delta \mathrm{IEC}}$ mice crossed with an IL-10-green fluorescent protein (GFP) reporter line (Vert-X) exhibited similar concentrations of IL-10 (fig. S8A) and/or frequencies of reporter ${ }^{+}$LP B cells (fig. S8B) compared with those of their respective littermate controls.

We examined if luminal $\operatorname{IgA}$ secretion was required for the protective role observed by generating polymeric immunoglobulin receptor (Pigr) and $X b p 1$ double-deficient mice $\left(P_{i g r^{-}}{ }^{-} X b p 1^{\Delta \mathrm{IEC}}\right)$, which are unable to transport IgA and IgM across the IEC (18). Pigr ${ }^{-1-}$ $X b p 1^{\Delta \mathrm{IEC}}$ mice showed an increase of $\mathrm{LP}_{\mathrm{IgA}}{ }^{+}$plasma cells similar to $X b p 1^{\Delta \mathrm{IEC}}$ animals (Fig. 1, M and N) but still developed severe inflammation of the proximal SI (Fig. $1 \mathrm{O}$ and fig. S5, histology scores and H\&E, respectively). This phenocopied $I g h a^{-1-} X b p 1^{\Delta \mathrm{IEC}}$ and $\mu M T X b p 1^{\Delta \mathrm{IEC}}$ animals and indicated a protective role for $\operatorname{sIgA}$ in this model. Although $X b p 1^{\Delta \mathrm{IEC}}$ animals exhibited increased IgA coating of fecal bacteria compared with $X b p 1^{\mathrm{fl} / \mathrm{fl}}$ controls (Fig. 1P), IgA-SEQ (6) revealed no major differences between $X b p 1^{\Delta \mathrm{IEC}}$ and $X b p I^{\mathrm{fl} / \mathrm{fl}}$ mice in the taxa-specific coating of commensal bacteria with IgA, suggesting a specific IgA-targeted microbe was not responsible (fig. S9). 
Intestinal IgA ${ }^{+}$plasma cells can differentiate via $\mathrm{T}$ cell-dependent (TD) and $\mathrm{T}$ cellindependent (TI) pathways $(19,20)$. Although we observed a small increase in germinal center B cells (B220 $\left.{ }^{+} \mathrm{CD} 19^{+} \mathrm{CD} 95^{+} \mathrm{GL}^{+}\right)$in Peyer's patches (PP) of $X b p 1^{\mathrm{AIEC}}$ mice compared with that in PP of $X b p 1^{\mathrm{fl} / \mathrm{fl}}$ controls (Fig. 2A), TD pathways were not involved in the IgA induction. First, $\mathrm{T}$ follicular helper $\left(\mathrm{T}_{\mathrm{FH}}\right)$ cell percentages $\left(\mathrm{CD} 3{ }^{+} \mathrm{CD} 4^{+} \mathrm{ICOS}\right.$ ${ }^{+} \mathrm{PD}-1^{\mathrm{hi}} \mathrm{CXCL} 5^{\mathrm{hi}}$ ) (7) in the PP and mesenteric lymph nodes (MLN) of $X b p 1^{\mathrm{AIEC}}$ mice were similar to those in $X b p 1^{\mathrm{fl} / \mathrm{fl}}$ controls (Fig. 2B). Second, T cell receptor $\beta$-deficient $T C R \beta^{-1-}$ $X b p 1^{\Delta \mathrm{IEC}}$ mice exhibited increased SI LP IgA ${ }^{+}$plasma cell numbers (Fig. $2 \mathrm{C}$ ) without changes in LP $\gamma \delta 5$ T cells (fig. S10) compared with $T C R \beta^{-1-} X b p I^{\mathrm{fl} l / f l}$ controls. Finally, PPdeficient (PPdef) $X b p 1^{\Delta I E C}$ mice (21) continued to exhibit increased SI LP IgA ${ }^{+}$plasma cells (Fig. 2D) without proximal extension of SI inflammation (fig. S11) compared with $\mathrm{PP}^{\mathrm{def}} X b p I^{\mathrm{f} / \mathrm{fl}}$ littermate controls.

By contrast, we observed increased percentages and numbers of $\mathrm{B} 1 \mathrm{~b}\left(\mathrm{CD} 5^{-} \mathrm{CD} 19^{+} \mathrm{CD} 23^{-}\right.$ $\mathrm{CD}^{+} 3^{+}$, but not B1a $\left(\mathrm{CD}^{+} \mathrm{CD} 19^{+} \mathrm{CD} 23^{-} \mathrm{CD} 43^{+}\right)$, cells in the peritoneal cavities of $X b p 1^{\Delta \mathrm{IEC}}$ mice compared with those in $X b p I^{\mathrm{fl} / \mathrm{fl}}$ littermate controls (Fig. 2, E and F). B1 cells associated with TI pathways emerge and migrate from there to the intestine, giving rise to polyreactive IgA-producing plasma cells in the SI $(19,22,23)$. As this suggested a transmissible factor, we conducted parabiosis experiments in which $\mathrm{CD} 45.1^{+}$wild-type (WT) mice were joined to CD45.2 $X b p 1^{\mathrm{AIEC}}$ mice or $X b p 1^{\mathrm{fl} / \mathrm{fl}}$ controls (Fig. 2G). Three weeks after parabiosis, we observed $\sim 50 \%$ chimerism of blood T and B cells (Fig. $2 \mathrm{H}$ ). Consistent with their tissue-resident phenotype, peritoneal B1 cells exhibited $\sim 20 \%$ chimerism (Fig. 2H). The peritoneal B1 cell compartments of WT CD45.1 mice showed increased numbers of $\mathrm{CD} 45.1^{+} \mathrm{B} 1 \mathrm{~b}$ cells in animals joined to $\mathrm{CD} 45.2^{+} X b p 1^{\mathrm{AIEC}}$ mice compared with those joined to CD45.2 $2^{+} X b p I^{\mathrm{fl} / \mathrm{fl}}$ controls (Fig. 2I and gating strategy in fig. S12). There were also more CD $45.1^{+} \mathrm{IgA}{ }^{+}$plasma cells in the SI LP of CD45.2 $2^{+} X b p 1^{\Delta \mathrm{IEC}}$ mice than in that of CD45.2 $X b p 1^{\text {fl/fl }}$ controls, which lacked ER stress in their intestinal epithelium (Fig. 2J). By contrast, CD45.1 ${ }^{+} \mathrm{IgA}{ }^{+}$plasma cells in the $\mathrm{LP}$ of CD45.1 $1^{+}$animals were not increased (Fig. 2K). Notably, there were no significant changes in SI Tnfsf13 (Apri), Tnfsf13b (Baff), Ccl25, Ccl28, and Cxcl13 expression (fig. S13A) or thymic stromal lymphopoietin protein levels (fig. S13B), which have been implicated in TI IgA class switching and/or plasma-cell recruitment (24).

Germ-free (GF) $X b p 1^{\Delta \mathrm{IEC}}$ mice, compared with GF $X b p I^{\mathrm{fl} / \mathrm{fl}}$ controls, also exhibited increased numbers of SI IgA $\mathrm{A}^{+}$plasma cells (Fig. 3, A and B), basal plasmacytosis (Fig. 3B), higher frequencies and numbers of peritoneal B1b cells (Fig. 3, C and D), and an increased proportion of IgA ${ }^{+}$cells within the SI LP B1b-like cell compartment $\left(\mathrm{CD}{ }^{-} \mathrm{CD} 19^{+} \mathrm{CD} 43^{+}\right)$ (Fig. 3E). They also showed heightened IEC ER stress (fig. S14, A to C) without spontaneous enteritis (5) and few SI epithelial apoptotic events compared with specific pathogen-free (SPF) $X b p 1^{\mathrm{AIEC}}$ mice (fig. S14D). GF $X b p 1^{\Delta \mathrm{IEC}}$ mouse colons also showed increased numbers of IgA ${ }^{+}$plasma cells (fig. S15A) and higher concentrations of tissue $\operatorname{IgA}$ (fig. S15B) than colons of littermate controls. However, colonic IgA ${ }^{+}$plasma cell numbers and IgA tissue concentrations in SPF $X b p 1^{\mathrm{\Delta IEC}}$ and $X b p 1^{\text {fl/fl }}$ mice were similar (fig. S15, A and $\mathrm{B}$ ), suggesting that high levels of TD IgA production in the colon mask the TI ER stress-induced IgA response under SPF conditions (25). 
Thus, the increase in $\operatorname{IgA}^{+}$plasma cells was not restricted to the SI nor dependent on apoptosis, microbiota, or a proinflammatory milieu but, rather, was due to IEC ER stressdriven recruitment of TI peritoneal B1b cells. Indeed, although single-cell RNA sequencing of the peritoneal lavage of GF $X b p 1^{\mathrm{AIEC}}$ mice and $X b p 1^{\mathrm{fl} / \mathrm{fl}}$ controls identified heterogeneous populations of peritoneal myeloid, B cell, and T cell subsets (Fig. 3, F to H, and fig. S16, A and B), the only peritoneal cell type demonstrating a major expansion in the context of IEC ER stress was a cluster containing a B1b-like transcriptional signature (cluster 2; Fig. 3, F to H, and fig. S16, A and B). Flow cytometry confirmed the absence of peritoneal myeloid or T cell alterations (fig. S17) or changes in SI LP myeloid cell populations (fig. S18). Peritoneal B1b cells from GF $X b p 1^{\Delta \mathrm{IEC}}$ mice were also transcriptionally distinct. Differential expression (Fig. 3I) and gene set enrichment analysis (GSEA; Fig. 3J) of purified B1b cells from GF $X b p 1^{\mathrm{AIEC}}$ mice showed the up-regulation of genes involved in protein biosynthesis, oxidative phosphorylation, and Myc signaling-which is critical for B cell activation (26)-compared with B1b cells of GF littermate controls. By contrast, cell adhesion gene sets were down-regulated in line with the increased ability of these B1b cells to egress from the peritoneal cavity and home to ER-stressed SI epithelium (Fig. 3J).

Taking advantage of the increased circulating IgA concentrations present in GF $X b p 1^{\Delta \mathrm{IEC}}$ mice compared with those in GF $X b p 1^{\mathrm{fl} / \mathrm{fl}}$ mice (Fig. 3K), we functionally confirmed the B1 origin of the $\operatorname{Ig}$ A response by showing that the $\operatorname{IgA}$ derived from these animals efficiently coated fecal microbiota obtained from $\mu$ MT mice lacking immunoglobulins (Fig. 3L) and exhibited broad reactivity to endogenous and exogenous antigens (Fig. 3M) as expected (23, 25). Furthermore, analysis of variable-region usage and CDR3 clono-type sequences from the proximal and distal intestinal segments of GF animals demonstrated the existence of a similar Ig $\mathrm{A}^{+}$cell polyclonal repertoire (fig. S19, A to C) containing a limited CDR3 region mutational load (fig. S19D) regardless of genotype.

Lastly, mice with a conditional deletion of autophagy related 16-like 1 in IECs (Atg1611 ${ }^{\mathrm{IEC}}$ ) exhibit SI IEC ER stress without histopathologic signs of inflammation (5, 11). These animals exhibited increased numbers of SI LP IgA $\mathrm{A}^{+}$cells (Fig. 4A) and a specific increase in peritoneal B1b cells (Fig. 4B). Similarly, SI biopsies of healthy human subjects homozygous for the hypomorphic $A T G 16 L 1^{\mathrm{T} 300 \mathrm{~A}}$ variant, who are known to exhibit increased ER stress (27), showed higher numbers of $\mathrm{LP} \mathrm{IgA}^{+}$cells than both non-carriers and heterozygous subjects (Fig. 4C).

Thus, the secretion of $\operatorname{IgA}$ into the lumen and resultant innate-like polyreactive responses protect ER-stressed mucosa in a pathway under the control of IEC ER stress. This occurs independently of either microbes or inflammation, making it a self-contained, host-derived response. This response is TI, peritoneal B1b cell-derived, and under the control of an unknown transmissible factor that emerges from ER stress in the IEC and is communicated to the peritoneal cavity, revealing a tight link between these two anatomic sites. In the absence of IgA or its secretion, spontaneous enteritis emerges. We propose that this homeostatic function of epithelial ER stress is a beneficial "eustress" response that is functionally opposed to its well-described involvement in proinflammatory pathways. 


\section{Supplementary Material}

Refer to Web version on PubMed Central for supplementary material.

\section{ACKNOWLEDGMENTS}

The authors would like to thank K. Cadwell (NYU), T. Chiba (Kyoto University), and J. Mestecky (University of Alabama) for useful discussions.

Funding: This work was supported by NIH grants DK044319, DK051362, DK053056, and DK088199; the Harvard Digestive Diseases Center (HDDC) DK034854 (R.S.B.); the Wellcome Trust Senior Investigator Award 106260/Z/14/Z, Evelyn Trust 13/27 (A.K.); the H0RIZ0N2020/European Research Council Consolidator Grant 648889 (A.K.); the National Institute for Health Research Cambridge BRC Cell Phenotyping Hub (A.K.); Rubicon grant 825.13.012, Netherlands Organization for Scientific Research (J.G.); JSPS KAKENHI grant number 2689323 and 16K19162, Japan Foundation for Applied Enzymology (S.H.); NIH and NIAID grants R01AI24998 and R21AI117220 (M.E.C); Deutsche Forschungsgemeinschaft grant KR 4749/1-1 (N.K.); NIH NCI grant R01 CA238039 (K.W.W.); DFG ExC Precision Medicine in Chronic Inflammation, H2020 SYSCID \#733100 and DFG CRC1182, C2 (P.R.); and Pediatric Scientist Development Program K12- HD000850 (J.D.M.). The Howard Hughes Medical Institute supports R.A.F.

\section{REFERENCES AND NOTES}

1. Grootjans J, Kaser A, Kaufman RJ, Blumberg RS, Nat. Rev. Immunol. 16, 469-484 (2016). [PubMed: 27346803]

2. Bogaert S et al., PLOS ONE 6, e25589 (2011).

3. Kaser A et al., Cell 134, 743-756 (2008). [PubMed: 18775308]

4. Tréton X et al., Gastroenterology 141, 1024-1035 (2011). [PubMed: 21699776]

5. Adolph TE et al., Nature 503, 272-276 (2013). [PubMed: 24089213]

6. Palm NW et al., Cell 158, 1000-1010 (2014). [PubMed: 25171403]

7. Pabst O, Nat. Rev. Immunol. 12, 821-832 (2012). [PubMed: 23103985]

8. Gutzeit C, Magri G, Cerutti A, Immunol. Rev. 260, 76-85 (2014). [PubMed: 24942683]

9. Kaser A, Blumberg RS, Cell Res. 18, 1160-1162 (2008). [PubMed: 19043437]

10. Bitton A et al., Gastroenterology 120, 13-20 (2001). [PubMed: 11208709]

11. Tschurtschenthaler M et al., J. Exp. Med. 214, 401-422 (2017). [PubMed: 28082357]

12. Luo S, Mao C, Lee B, Lee AS, Mol. Cell. Biol. 26, 5688-5697 (2006). [PubMed: 16847323]

13. Cao SS et al., Gastroenterology 144, 989-1000.e6 (2013). [PubMed: 23336977]

14. Mellander L, Björkander J, Carlsson B, Hanson LÅ, J. Clin. Immunol. 6, 284-291 (1986).

[PubMed: 3489000]

15. Fadlallah J et al., Sci. Transl. Med 10, eaan1217 (2018).

16. Magri G et al., Immunity 47, 118-134.e8 (2017). [PubMed: 28709802]

17. Mauri C, Menon M, J. Clin. Invest. 127, 772-779 (2017). [PubMed: 28248202]

18. Johansen FE et al., J. Exp. Med. 190, 915-922 (1999). [PubMed: 10510081]

19. Macpherson AJ et al., Science 288, 2222-2226 (2000). [PubMed: 10864873]

20. Macpherson AJ, McCoy KD, Johansen FE, Brandtzaeg P, Mucosal Immunol. 1, 11-22 (2008). [PubMed: 19079156]

21. Yoshida H et al., Int. Immunol. 11, 643-655 (1999). [PubMed: 10330270]

22. Baumgarth N, Nat. Rev. Immunol. 11, 34-46 (2011). [PubMed: 21151033]

23. Bunker JJ et al., Immunity 43, 541-553 (2015). [PubMed: 26320660]

24. Cerutti A, Nat. Rev. Immunol. 8, 421-434 (2008). [PubMed: 18483500]

25. Bunker JJ et al., Science 358, eaan6619 (2017).

26. de Alboran IM et al., Immunity 14, 45-55 (2001). [PubMed: 11163229]

27. Deuring JJ et al., Gut 63, 1081-1091 (2014). [PubMed: 23964099] 


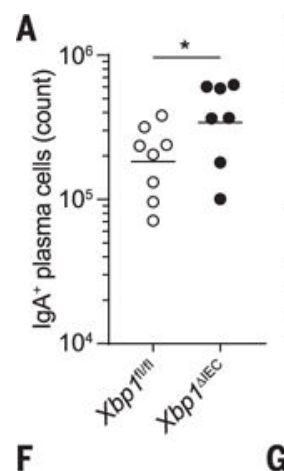

$\mathbf{F}$
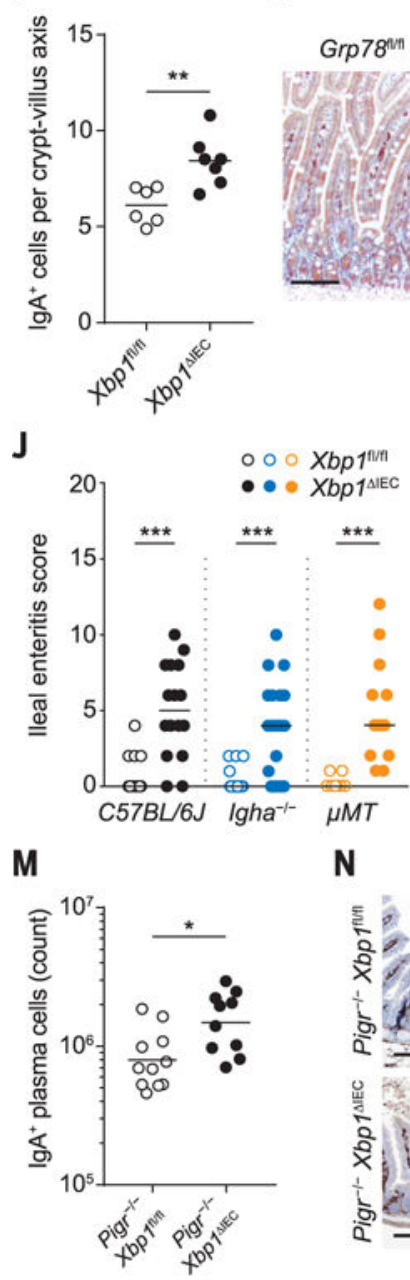

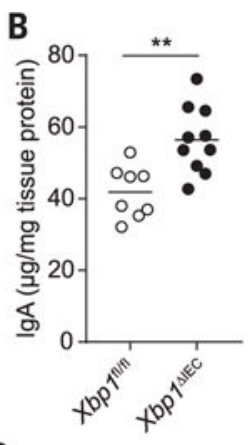

G

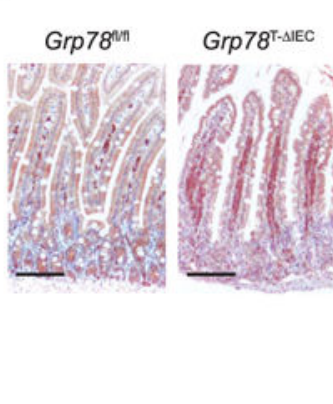

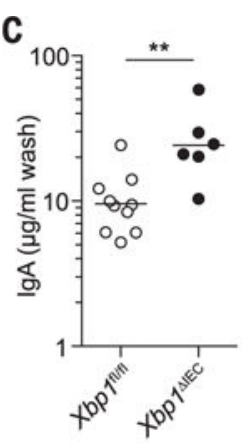

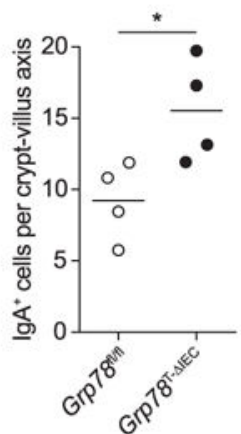

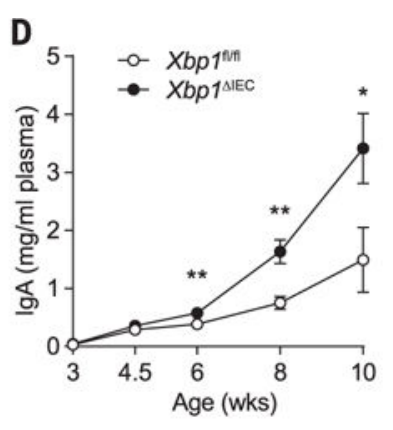

H

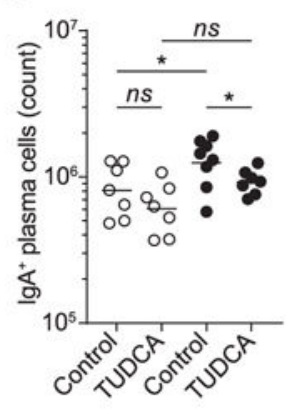

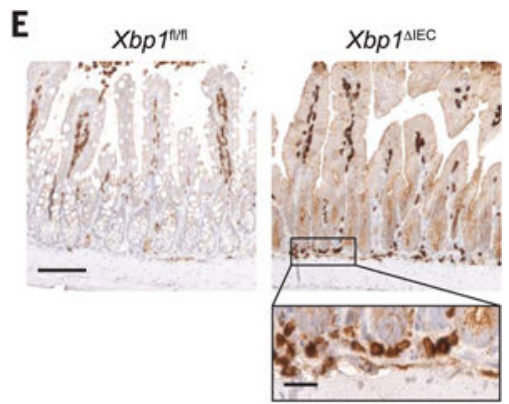

I

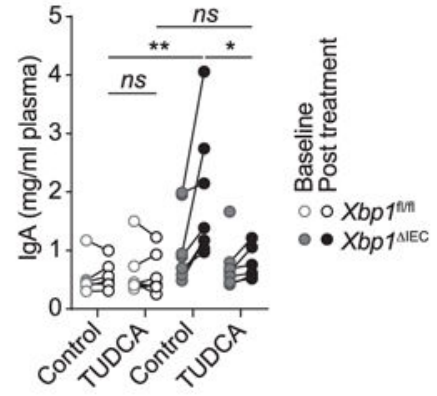

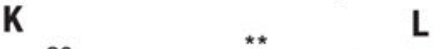

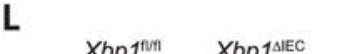

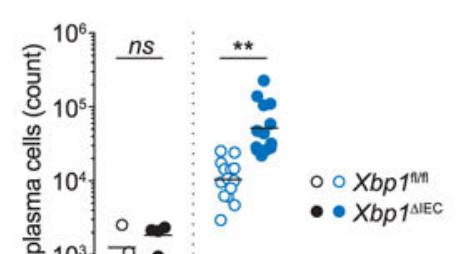

Fig. 1. Intestinal epithelial ER stress induces a protective IgA response.

(A) Absolute counts of SI LP IgA ${ }^{+}$plasma cells in $X b p 1^{\Delta \mathrm{IEC}}$ mice and $X b p I^{\mathrm{fl} / \mathrm{fl}}$ controls at 10 weeks of age ( $n=7$ or 8 ). (B) Ileal tissue IgA normalized by total soluble tissue protein ( $n=8$ to 10). (C) IgA concentration in SI washes ( $n=6$ to 10). (D) Circulating IgA concentration ( $n=6$ to 10 for each age). ( $\mathbf{E}$ and $\mathbf{F}$ ) Representative IHC images (E) and quantification (F) of $\mathrm{LP} \mathrm{IgA}^{+}$cells (brown) along 250 ileal crypt-villus axes ( $n=6$ or 7). Magnified area in (E) depicts basal plasmacytosis. (G) Representative IHC images and quantification of SI LP IgA ${ }^{+}$cells (red) in Grp $78^{\mathrm{T}-\Delta \mathrm{IEC}}$ mice and $G r p 78^{\mathrm{fl} / \mathrm{fl}}$ controls after 3 
days of tamoxifen treatment $(n=4)$. ( $\mathbf{H}$ and $\mathbf{I})$ Absolute counts of SI LP IgA ${ }^{+}$plasma cells (H) and circulating IgA concentrations (I) of the indicated genotypes, treated with either TUDCA $(2 \mathrm{mg} / \mathrm{ml})$ in the drinking water or plain water (control) for two weeks ( $n=7$ or 8 ). $(\mathbf{J}$ and $\mathbf{K}$ ) Enteritis scores of ileal $(\mathrm{J})$ and jejunal $(\mathrm{K})$ sections of indicated genotypes ( $n=4$ to 26). (L) Representative plots, frequencies, and absolute counts of SI $\mathrm{LP} \mathrm{IgM}^{+}$plasma cells (gated on $\mathrm{CD}^{4} 5^{+} \mathrm{CD}^{-}$lymphocytes) of the indicated genotypes ( $n=3$ to 14 ). ( $\mathbf{M}$ to $\mathbf{O}$ ) Absolute flow cytometric counts of SI LP IgA ${ }^{+}$plasma cells (M), representative IHC images and quantification of $\mathrm{IgA}^{+}$cells in ileal sections (N), and enteritis scores (O) of $\mathrm{Pigr}^{-1}-$ $X b p 1^{\Delta I E C}$ mice and $P_{i g r^{-}-} X b p 1^{f l / f 1}$ controls ( $n=9$ to 18). (P) Frequencies of IgA-coated fecal bacteria from the indicated genotypes, as determined by flow cytometry ( $n=2$ to 20 ). B6 indicates a C57BL/6J background. Scale bars indicate $100 \mu \mathrm{m}$ (low magnification) or 20 $\mu \mathrm{mm}$ [magnified view in (E)]. Symbols represent individual animals. Bars represent arithmetic means $[(\mathrm{B}),(\mathrm{D}),(\mathrm{F}),(\mathrm{G}),(\mathrm{N})$, and $(\mathrm{P})]$, medians $[(\mathrm{J}),(\mathrm{K})$, and $(\mathrm{O})]$, or geometric means $[(\mathrm{A}),(\mathrm{C}),(\mathrm{H}),(\mathrm{L})$, and $(\mathrm{M})]$. Error bars indicate SEM. Data are representative of three $[(\mathrm{A})$ and $(\mathrm{B})]$ independent experiments or were compiled from two $(\mathrm{M})$ or three $[(\mathrm{L})$ and (P)] experiments. $P$ values were calculated by unpaired Student's $t$ test [(A) to (D), (F), $(\mathrm{G}),(\mathrm{L})$ to $(\mathrm{N})$, and $(\mathrm{P})]$, Kruskal-Wallis test with Dunn's post-test [(J) and $(\mathrm{K})]$, MannWhitney $U$ rank sum test (O), or two-way analysis of variance (ANOVA) with Fisher's leastsignificant difference (LSD) method and two-stage step-up method of Benjamini, Krieger, and Yekutieli to control the false discovery rate $[(\mathrm{H})$ and $(\mathrm{I})] . * P<0.05 ; * * P<0.01 ; * * * P$ $<0.001$; ns, not significant. 

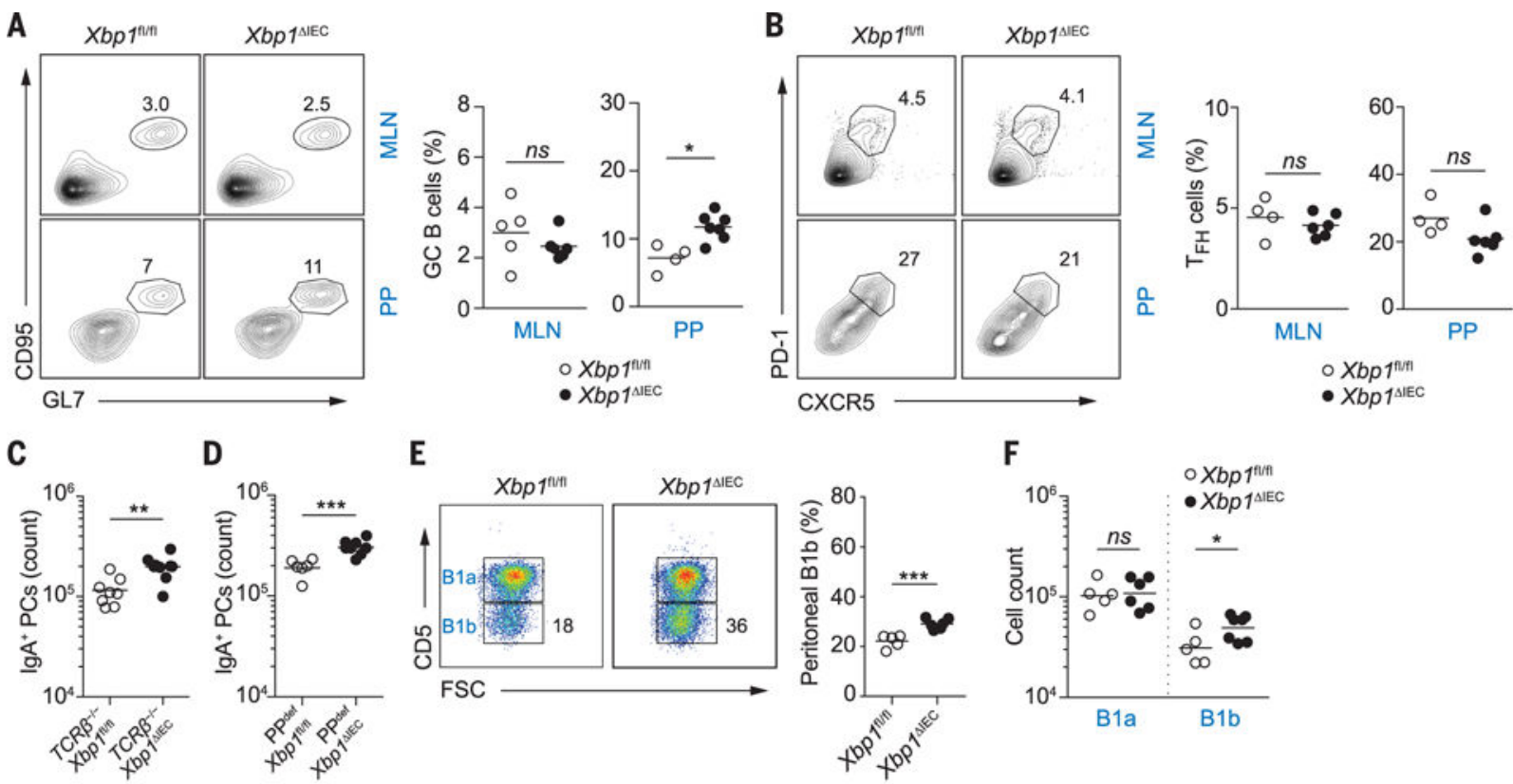

$\mathbf{E}$
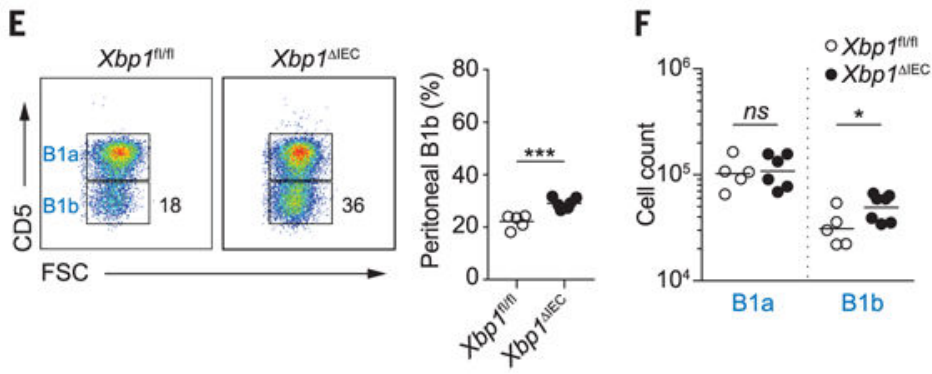

\section{G} $\begin{array}{cc}\text { CD45.2 Xbp 1m } & \text { CD } 45.2 \times b p 1^{\text {NeC }}\end{array}$
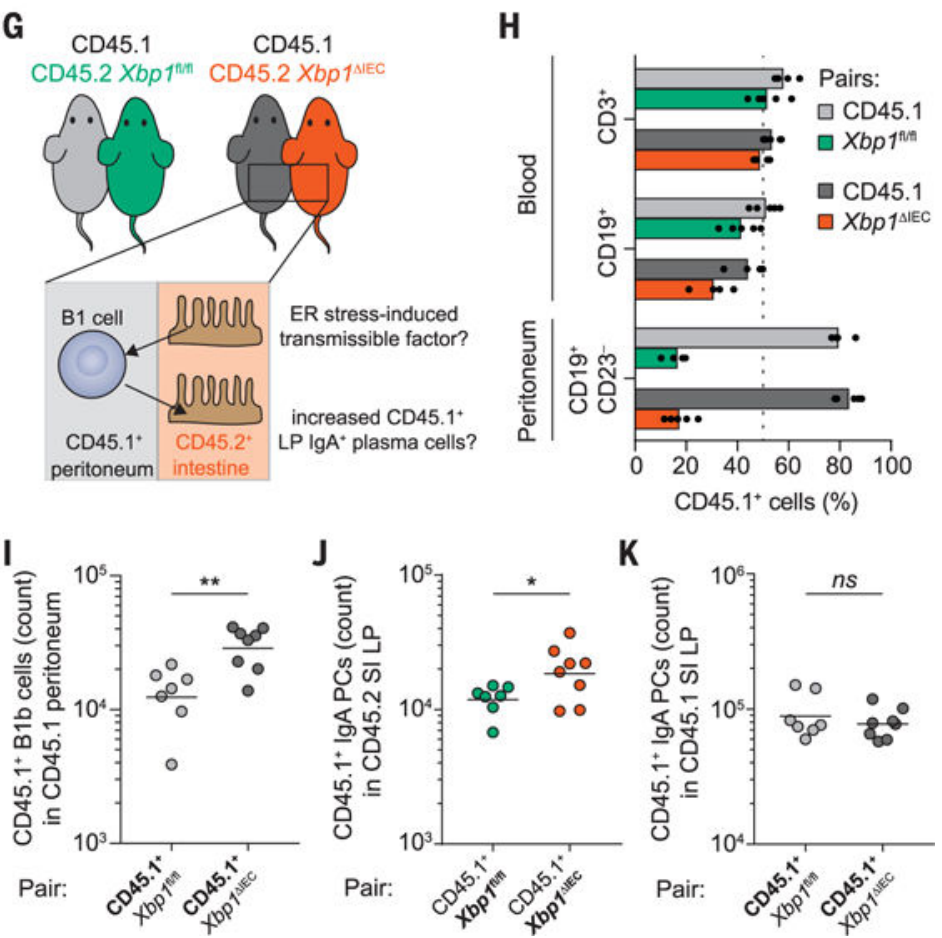

Fig. 2. ER stress-induced IgA is PP- and T cell-independent and involves recruitment of peritoneal B1b cells by a transmissible factor.

(A) Representative plots and percentages of germinal center (GC) B cells (gated on CD19 ${ }^{+}$ lymphocytes) in MLN and PP of $X b p 1^{\Delta \mathrm{IEC}}$ mice and $X b p 1^{\mathrm{fl} / \mathrm{fl}}$ controls $(n=4$ to 7$)$. (B) Representative plots and percentages of MLN and PP $\mathrm{T}_{\mathrm{FH}}$ cells (gated on $\mathrm{CD} 3{ }^{+} \mathrm{CD}^{+}$ lymphocytes, $n=4$ to 6). (C) Absolute counts of SI LP IgA ${ }^{+}$plasma cells (PCs) in TCR $\beta^{-1-}$ $X b p 1^{\Delta \mathrm{IEC}}$ mice and $T C R \beta^{-1-} X b p 1^{\mathrm{fl} / \mathrm{fl}}$ controls $(n=8$ or 9). (D) Absolute counts of SI LP $\mathrm{IgA}^{+}$plasma cells in PP-deficient $X b p 1^{\Delta \mathrm{IEC}}$ mice and $X b p 1^{\mathrm{fl} / \mathrm{fl}}$ controls $(n=6$ to 8$)$. (E and 
F) Representative plots, percentages, and absolute counts of peritoneal B1a and B1b cells in $X b p 1^{\mathrm{AIEC}}$ mice and $X b p 1^{\mathrm{fl} / \mathrm{fl}}$ controls $(n=5$ to 7$)$. FSC, forward scatter. (G) Schematic representation of the parabiosis experiment $(n=7$ or 8 pairs per genotype). (H) Frequencies of CD $45.1^{+}$circulating lymphocytes and CD $45.1^{+}$peritoneal B1 cells 3 weeks after parabiotic surgery. The dotted line indicates 50\% chimerism. (I) Absolute numbers of CD $45.1^{+} \mathrm{B} 1 \mathrm{~b}$ cells in peritoneal cavities of CD45.1 animals conjoined with either Xbp $1^{\mathrm{fl} / \mathrm{fl}}$ or $X b p 1^{1 \mathrm{AEC}}$ mice. ( $\mathbf{J}$ and $\left.\mathbf{K}\right)$ Absolute numbers of SI LP CD45.1 $1^{+} \operatorname{IgA}+$ plasma cells in parabiotic $X b p I^{\mathrm{fl} / \mathrm{fl}}$ and $X b p 1^{\Delta \mathrm{IEC}}$ mice $(\mathrm{J})$ and in $\mathrm{CD} 45.1$ parabionts conjoined with either $X b p 1^{\mathrm{fl} / f \mathrm{l} l}$ or $X b p 1^{\Delta \mathrm{IEC}}$ mice (K). Symbols represent individual animals. Bars represent arithmetic means [(A), (B), (E), and (H)] or geometric means [(C), (D), (F), and (I) to (K)]. Data are representative of three experiments $[(\mathrm{E})$ and $(\mathrm{F})]$ or were pooled from two experiments [(C), (D), and (G) to (K)]. $P$ values were calculated by unpaired Student's $t$ test. $* P<0.05 ; * * P<0.01 ; * * * P<0.001 ;$ ns, not significant. 
A

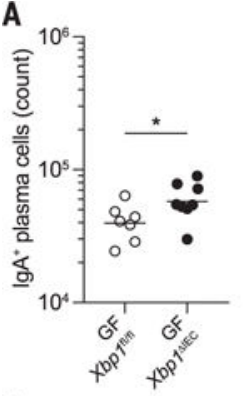

E

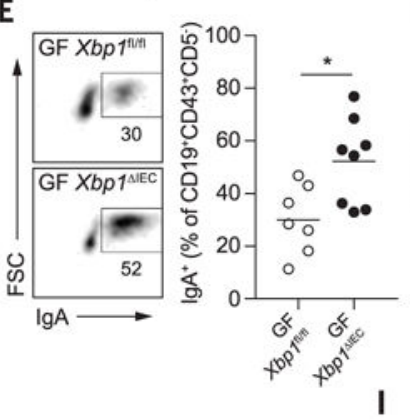

B

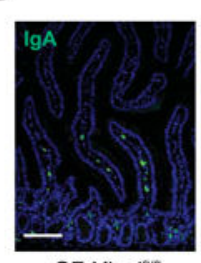

GF Xbp 1 1 nn

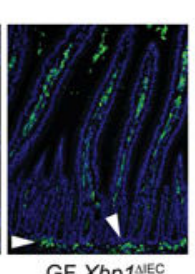

GF Xbp ${ }^{\mathrm{NEC}}$
G
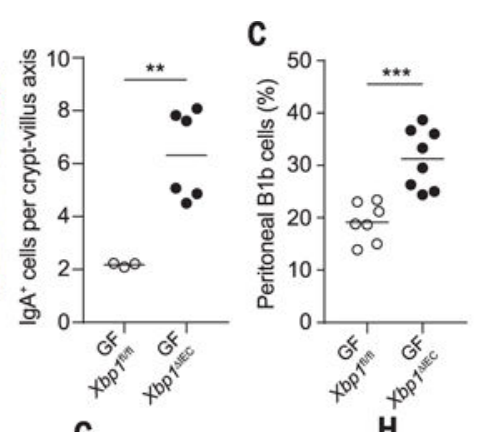

$\mathbf{F}$
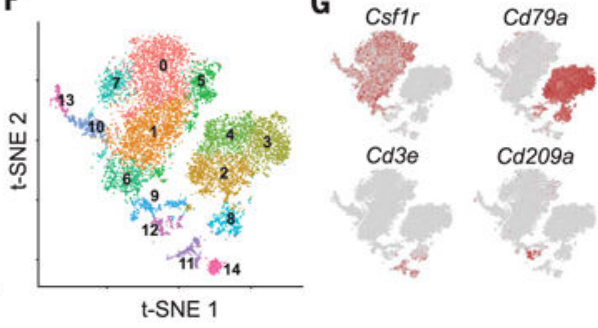

H
D

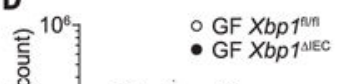

**
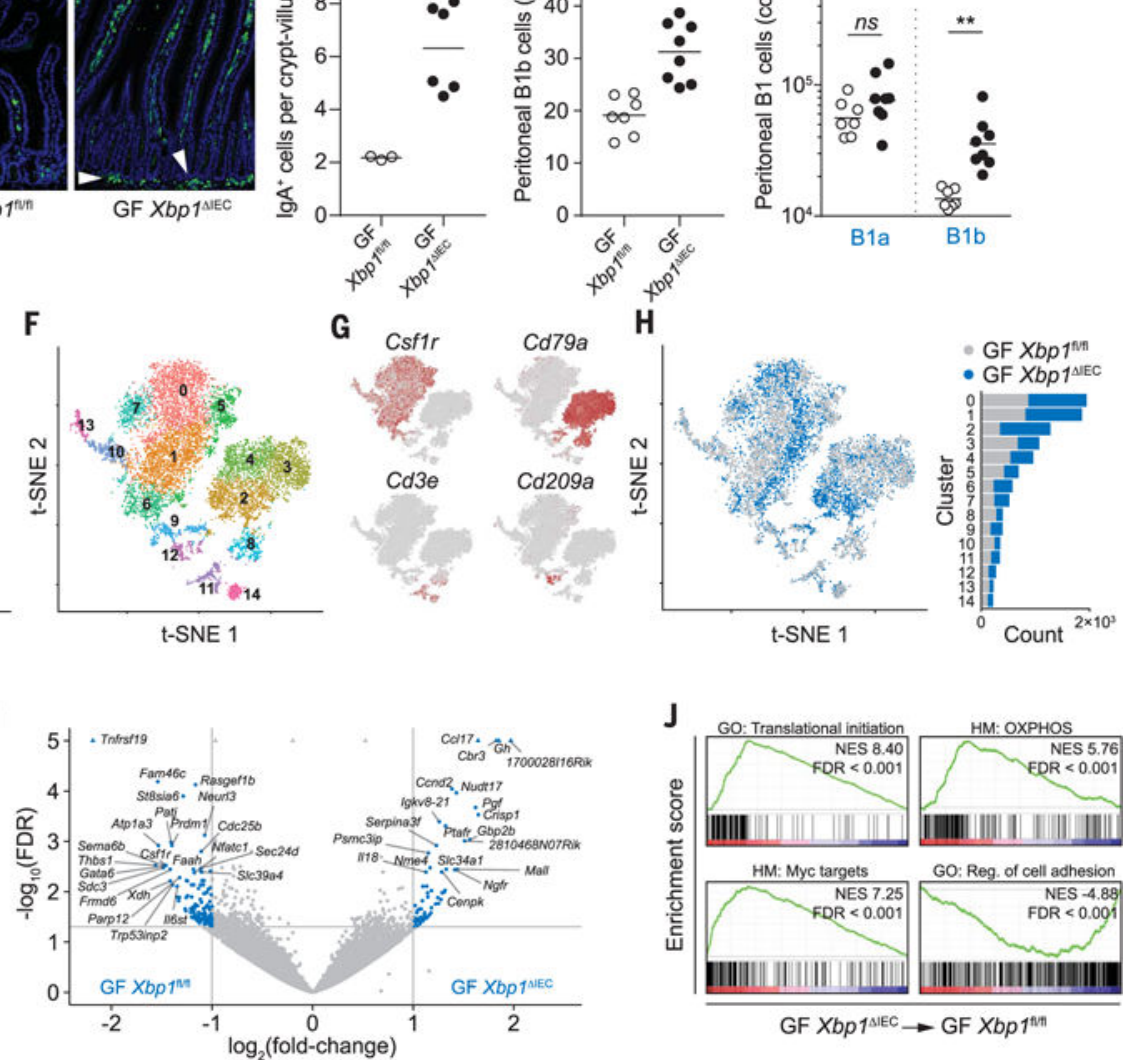

$\mathrm{GF} X \mathrm{Xbp} 1^{\mathrm{AIEC}} \rightarrow \mathrm{GF} X \mathrm{Xbp} 1^{\text {thI }}$

K

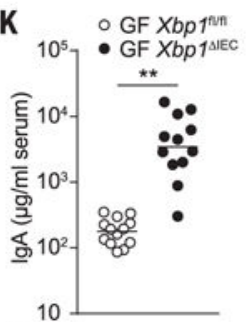

L

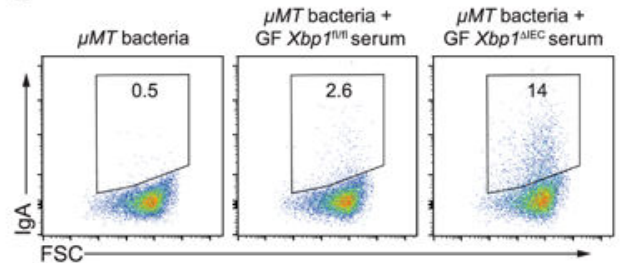

M
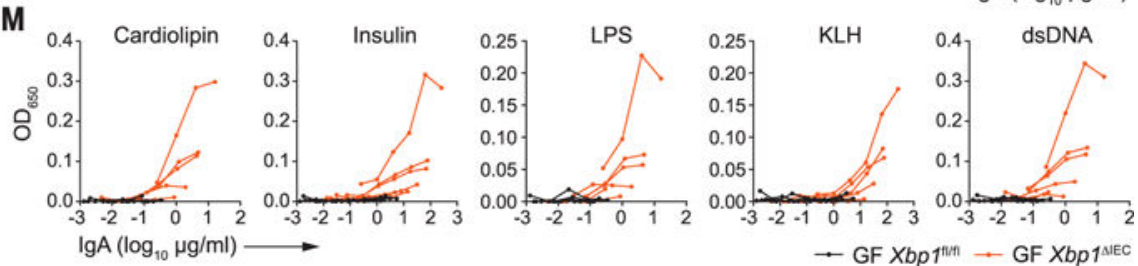

Fig. 3. Epithelial ER stress-derived IgA is microbiota- and inflammationindependent and polyreactive in nature.

(A) Absolute counts of SI LP IgA ${ }^{+}$plasma cells in GF $X b p 1^{\Delta \mathrm{IEC}}$ mice and $X b p 1^{\mathrm{fl} / \mathrm{fl}}$ controls ( $n=7$ or 8). (B) Representative immunofluorescence images and quantification of $\mathrm{LP} \mathrm{IgA}^{+}$ cells (green) along $\geq 50$ ileal crypt-villus axes ( $n=3$ to 6 ). Nuclei are counterstained with $4^{\prime}$, 6-diamidino-2-phenylindole (DAPI) (blue). Arrows indicate basal plasmacytosis. Scale bar, $100 \mu \mathrm{m}$. (C and D) Frequencies (percentage of CD19+CD23 ${ }^{-} \mathrm{CD} 43^{+}$cells) (C) and absolute flow cytometric counts of peritoneal B1a and B1b cells (D) $(n=7$ or 8). (E) Representative 
plots (gated on $\mathrm{CD}^{-} \mathrm{CD} 19^{+} \mathrm{CD} 43^{+}$lymphocytes) and frequencies of $\mathrm{IgA}^{+} \mathrm{B} 1 \mathrm{~b}$-derived cells in SI LP of GF $X b p 1^{\Delta \mathrm{IEC}}$ mice and GF $X b p 1^{\mathrm{fl} / \mathrm{fl}}$ controls $(n=7$ or 8$)$. (F) t-Distributed stochastic neighbor embedding (t-SNE) plot depicting unsupervised clustering of single-cell transcriptomes $\left(n=11,104\right.$ cells) from peritoneal lavages of $X b p 1^{\Delta \mathrm{IEC}}$ mice and $X b p I^{\mathrm{fl} / \mathrm{fl}}$ controls (aligned datasets). Numbers and colors indicate clusters. (G) Expression levels of canonical markers for macrophages $(C s f 1 r)$, B cells $(C d 79 a)$, T cells (Cd3e), and peritoneal dendritic cells ( $C$ d209a) in t-SNE plot. (H)t-SNE plot as in (F) with cells colored by genotype. Bar graph depicts the number of cells within each cluster by genotype. (I) Volcano plot showing $\log _{2}$-transformed fold-change $(\log 2 \mathrm{FC})$ of gene expression in $\mathrm{B} 1 \mathrm{~b}$ cells from GF $X b p 1^{\Delta \mathrm{IEC}}$ mice compared with that in B1b cells from GF $X b p 1^{\mathrm{fl} / f \mathrm{l}}$ controls $(n=5$ to 7$)$. Differentially expressed genes [ $\log 2 \mathrm{FC} \geq 1$ or $\leq-1$; false discovery rate $(\mathrm{FDR})<0.05]$ are highlighted in blue. FDR values that are $<10^{-5}$ are plotted at $10^{-5}$ (triangles). (J) GSEA enrichment plots for selected gene sets. GO, gene ontology gene sets; HM, hallmark gene sets; OXPHOS, oxidative phosphorylation; NES, normalized enrichment score. (K) Circulating IgA concentrations in GF $X b p 1^{\Delta \mathrm{IEC}}$ mice and $X b p 1^{\mathrm{fl} / \mathrm{fl}}$ controls $(n=12$ or 13$)$. (L) Representative plots (gated on SYBR ${ }^{\text {hi }}$ events) and frequencies of IgA coating on fecal bacteria from $\mu M T$ mice that were incubated with sera from GF $X b p 1^{\Delta \mathrm{IEC}}$ mice or $X b p 1^{\mathrm{fl} / \mathrm{fl}}$ controls ( $n=4$ or 5). (M) Polyreactivity enzyme-linked immune-sorbent assay optical density at $650 \mathrm{~nm}\left(\mathrm{OD}_{650}\right)$ values of serum IgA from GF $X b p 1^{\Delta \mathrm{IEC}}$ mice or $X b p I^{\mathrm{fl} / \mathrm{fl}}$ controls ( $n=5$ or 6 ) against the indicated antigens. LPS, lipopolysaccharide; KLH, keyhole limpet hemocyanin; dsDNA, double-stranded DNA. Symbols or lines represent individual animals. Bars represent arithmetic means [(B), (C), and (E)] or geometric means [(A), (D), and $(\mathrm{K})]$. Data are representative of at least two independent experiments [(B) to (D), (L), and $(\mathrm{M})]$ or were pooled from two experiments $[(\mathrm{A}),(\mathrm{E})$, and $(\mathrm{K})]$. $P$ values were calculated by unpaired Student's t test. $* P<0.05 ; * * P<0.01 ; * * *<<0.001$; ns, not significant. 
A

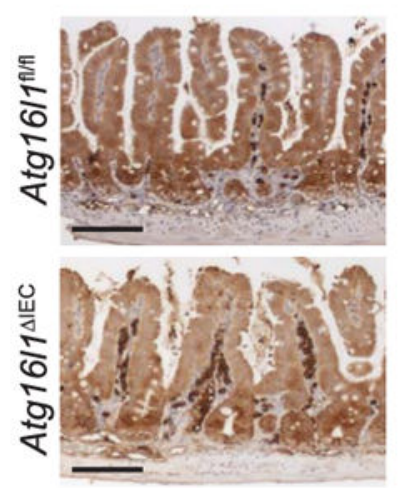

C

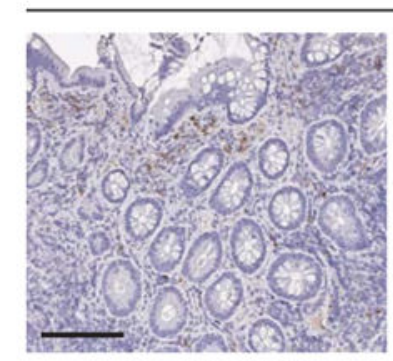

ATG16L1AA

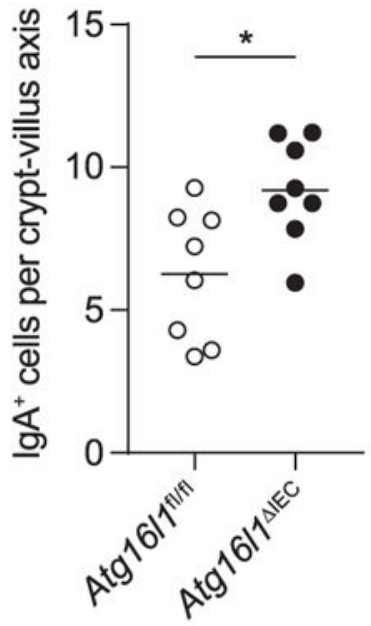

Healthy human subjects

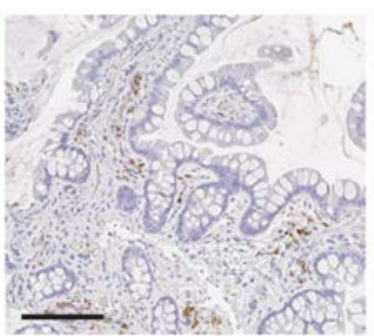

ATG16L $1^{A G}$
B

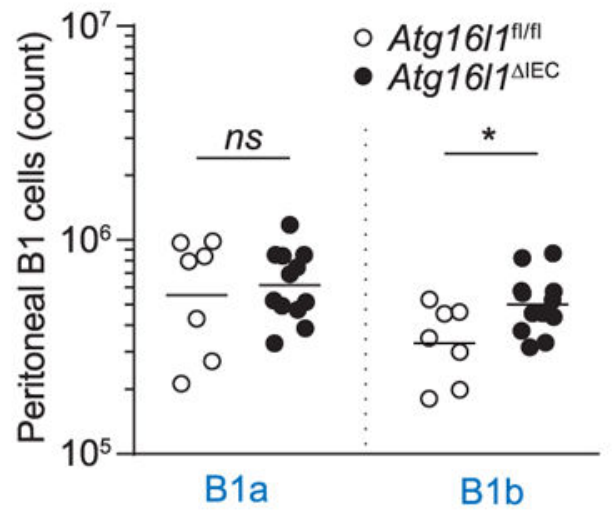

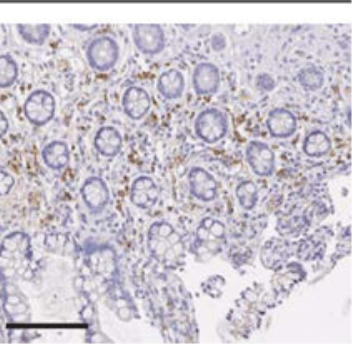

ATG16L1 ${ }^{\text {GG }}$

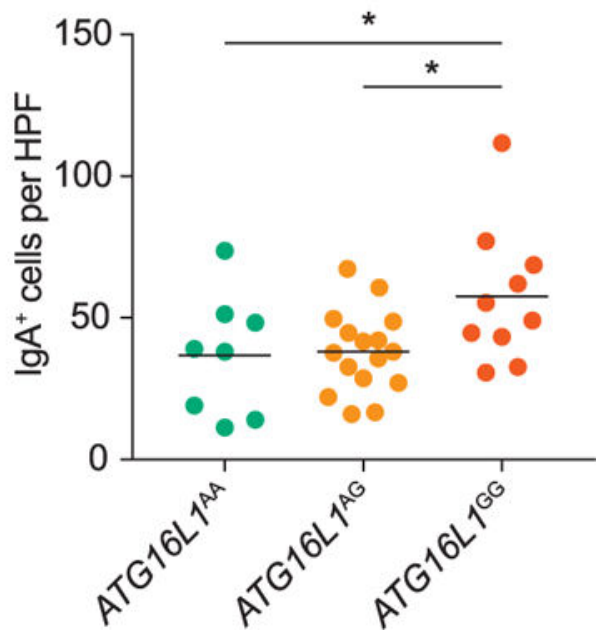

Fig. 4. Defective ATG16L1-dependent autophagy results in a peritoneal B1b response in mice and $\operatorname{IgA}$ induction in both mice and humans.

(A) Representative IHC images and quantification of LP $\operatorname{IgA}^{+}$cells (brown) along $\geq 50$ ileal crypt-villus axes of $A \operatorname{tg} 1611^{\Delta \mathrm{IEC}}$ mice and Atg1611 $1^{\mathrm{fl} / \mathrm{fl}}$ controls $(n=8)$. (B) Absolute counts

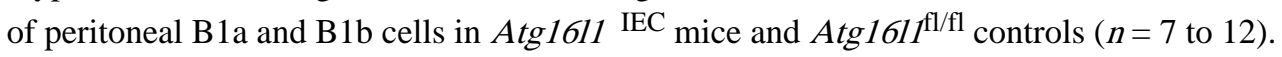
(C) Representative IHC images and quantification of $\operatorname{IgA}^{+}$cells (brown) in ileal biopsies of healthy human subjects, shown by $A T G 16 L 1$ genotype as indicated by AA, AG, and GG ( $n$ $=8$ to 16). Scale bars, $100 \mu \mathrm{m}$. HPF, high-power field. Symbols represent individual animals or human subjects. Bars represent arithmetic means $[(\mathrm{A})$ and $(\mathrm{C})]$ or geometric means (B). Data in (B) were pooled from two experiments. $P$ values were calculated by unpaired Student's $t$ test [(A) and (B)] or one-way ANOVA with Holm-Š́ídák test (C). ${ }^{*} P<0.05$; ns, not significant. 